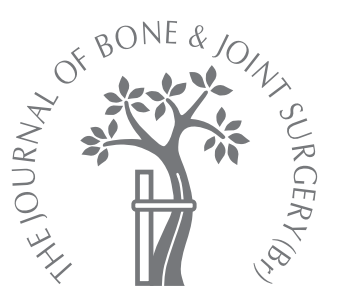

S. R. Bollen, D. Arvinte

From Bradford Teaching Hospital Trust, Bradford, England

S. R. Bollen, MB, FRCS, FRCSEd(Orth), FSEM(UK), Consultant Orthopaedic

Surgeon

D. Arvinte, MRCSEd, FEBOT, $\mathrm{FRCS}(T r$ \& Orth), Smith \& Nephew Knee Fellow Bradford Teaching Hospitals

Trust, Bradford Royal Infirmary, Duckworth Lane, Bradford BD9 6RJ, UK.

Correspondence should be sent to $\mathrm{Mr} \mathrm{S}$. R. Bollen; e-mail: srb@btinternet.com

C일 2008 British Editorial Society of Bone and Joint Surgery doi:10.1302/0301-620X.90B3. $20369 \$ 2.00$

$J$ Bone Joint Surg $[\mathrm{Br}]$ 2008;90-B:334-5.

Received 22 October 2007;

Accepted 20 November 2007

\section{Snapping pes syndrome}

\author{
A REPORT OF FOUR CASES
}

\begin{abstract}
We present a series of four patients with what we have termed the snapping pes syndrome. This is a painful clicking and catching experienced at the posteromedial corner of the knee when moving from flexion to extension. Clinical examination and real time ultrasound are the most useful diagnostic tools. If medical treatment is unsuccessful surgical excision of both the semitendinous and gracilis tendons is indicated for relief of persistent symptoms.
\end{abstract}

A snapping syndrome is a sudden jerk of movement felt whilst moving a joint. Joints which can be affected are the shoulder, ${ }^{1,2}$ elbow, ${ }^{3}$ wrist, ${ }^{4}$ hip, ${ }^{5}$ ankle, ${ }^{6}$ and knee. ${ }^{7-12}$ The snapping can be produced by a bony prominence, ${ }^{2}$ soft tissues outside or inside the joint, ${ }^{12}$ or a combination of these. The symptoms are often well tolerated and do not always require surgical treatment.

We are aware of only two studies in the English literature which report snapping of the knee caused by the semitendinosus or gracilis tendons. ${ }^{7,11}$

We present four patients with snapping of the tendons of semitendinosus and gracilis in a Caucasian population presenting over a sixyear period. We describe our method of treatment and suggest the name 'snapping pes syndrome' for this condition.

\section{Case reports}

Case 1. A 33-year-old man with a 17-year history of knee problems presented with a painful snapping sensation behind the posteromedial aspect of both knees when straightening them.

He had previously seen three orthopaedic surgeons with a specialist interest in knee surgery and undergone MR scanning, arthroscopy and an exploration and tendon release of the left knee with no improvement.

On examination the only finding was of a palpable clicking and catching over the posteromedial aspect of the knee when extending from the flexed position.

A further exploration of the left knee was undertaken. The problem seemed to be the semitendinosus tendon flicking around the semimembranosus tendon when moving the knee from flexion to extension. The semitendinosus tendon was excised, using a Linvatec tendon harvester (Linvatec UK Ltd, Swindon, United Kingdom) in the same way as harvesting the tendon for reconstruction of the an anterior cruciate ligament (ACL).

However, the symptoms continued. A dynamic real time ultrasound examination was performed, which showed that the gracilis tendon was still snapping around the posteromedial corner. The patient underwent a second procedure to remove the gracilis tendon, again using the same technique as for harvest of the hamstrings during ACL reconstruction.

This completely resolved the symptoms and the patient asked for the same procedure to be performed on his right knee. Again, this cured his complaints. A telephone follow-up confirmed that he is free of symptoms six years after surgery.

Case 2. A 36-year-old woman started to complain of pain over the medial side of her left knee approximately six months after increasing the level of her routine aerobic training. Medial pain was associated mainly with turning and twisting exercises. It was most severe at the end of the training session but persisted to the next day.

In the two months before she presented to our clinic, she started to feel a sensation of clicking as well as pain on the medial side of her left knee. The clicking could be felt during exercise, especially twisting but was most severe at the end of a training session.

Non-steroidal anti-inflammatory drugs and a course of physiotherapy had little effect. The pain on the medial side of the knee and painful snapping persisted when the knee was in a position of approximately $30^{\circ}$ of flexion during extension to flexion. 
The only physical findings were of some tenderness over the posteromedial aspect of the left knee, and on palpation a definite snap or click could be felt when moving the knee from flexion to extension, at approximately $30^{\circ}$ of flexion. The snap was accompanied by pain, with or without palpation over the area.

Radiographs of the left knee were normal. MRI showed no abnormality in relation to the medial hamstrings. Dynamic real-time ultrasound examination showed that the gracilis and semitendinosus tendons were flicking over the muscle belly of semimembranosus at approximately $30^{\circ}$ of flexion as the knee was moved from full flexion to extension and vice versa. No signs of tenosynovitis, pes anserinus bursitis or semimembranosus tendinosis were seen.

The patient was treated by excision of the semitendinosus and gracilis tendons as in ACL reconstruction through a $2 \mathrm{~cm}$ to $3 \mathrm{~cm}$ incision. After surgery the snapping/clicking disappeared. The patient returned to full activity by 12 weeks after operation and has remained symptom free.

Case 3. A 32-year-old, non-athletic woman, presented with a two-year history of painful clicking over the posteromedial aspect of the right knee. There was no precipitating incident. She had undergone conservative treatment with no improvement.

The clinical findings were identical to the two previous patients and she underwent the same surgical procedure with resolution of her symptoms.

Case 4. A 35-year-old male General Practitioner, who was a keen runner, presented with a 15 -month history of posteromedial pain and a snapping feeling over the posteromedial corner of the knee. As one of his friends was a radiologist, he had repeated MR scanning which showed semimembranosus tendonitis and a small Bakers cyst.

On examination there was a palpable and painful clicking over the posteromedial corner of the knee when moving from flexion to extension and he was referred back to the radiologist for examination with real time ultrasound, which seemed to show that the problem was 'jumping' of the semitendinosus tendon around the semimembranosus. After discussion, he agreed to have excision of this tendon only. This was performed in the standard fashion, but his symptoms persisted. He then underwent a second procedure to harvest the gracilis tendon and as a result the symptoms of painful clicking/snapping resolved.

\section{Discussion}

In the knee, the biceps, semitendinosus and gracilis tendons as well as intra-articular causes have been noted to produce snapping syndromes requiring surgical treatment. ${ }^{7-12}$ Two previous case reports ${ }^{7,11}$ have described the semitendinosus and gracilis tendons as a cause of snapping knee in the Asian population. However, to our knowledge this is the first time a series of snapping semitendinosus and gracilis tendons has been reported in Caucasians.

The diagnosis of this condition was mainly clinical in the paper by Lyu and Wu, ${ }^{11}$ but Bae and $\mathrm{Kwon}^{7}$ also used a pneumoarthrogram and ultrasound. In our opinion the most useful investigation in this syndrome is with real-time ultrasound carried out by an experienced musculoskeletal radiologist.

There was no evidence of valgus deformity, scissor gait or hyperextension of the knee in our patients, in comparison with the two Asian patients noted above. ${ }^{7,11}$ Surgical treatment in these patients required longer incisions $(10 \mathrm{~cm})$ as well as suturing of the tendinous stump to semimembranosus. In one paper ${ }^{11}$ only the semitendinosus tendon was excised.

Whilst removing the tendons may sound drastic, the senior author (SRB) performs this as day case surgery on a regular basis when performing reconstruction of the ACL. It produces little morbidity with an incision $2 \mathrm{~cm}$ to $3 \mathrm{~cm}$ in length. Hamstring harvest has been shown to cause no functional problems in the knee. ${ }^{13}$

In the two patients where excision of the semitendinosus tendon only was carried out, although this was the implicated tendon, there was no improvement of the clicking/ snapping. This disappeared only after both the semitendinosus and gracilis tendons were resected. No patient has reported a recurrence of symptoms.

No benefits in any form have been received or will be received from a commercial party related directly or indirectly to the subject of this article.

\section{References}

1. Bristow WR. A case of snapping shoulder. J Bone Joint Surg 1924;6:53-5.

2. Parsons TA. The snapping scapula and subscapular exostoses. J Bone Joint Surg [Br] 1973;55-B:345-9.

3. Rolfsen L. Snapping triceps tendon with ulnar neuritis: report on a case. Acta Orthop Scand 1970;41:74-6.

4. Istubo T, Uchiyama S, Takahara K, et al. Snapping wrist after surgery for carpal tunnel syndrome and trigger digit: a case report. J Hand Surg [Am] 2004;29:384-6.

5. Ilizaliturri VM Jr, Villalobos FE Jr, Chaidex PA, Valero FS, Aguilera JM. Internal snapping hip syndrome: treatment by endoscopic release of the iliopsoas tendon. Arthroscopy 2005;21:1375-80.

6. Alm A, Lamke LO, Liljedahl SO. Surgical treatment of dislocation of the peroneal tendons. Injury 1975;7:14-19.

7. Bae DK, Kwon OS. Snapping knee caused by the gracilis and semitendinosus tendon: a case report. Bull Hosp Joint Dis 1997:56:177-9.

8. Beals RK. The snapping knee of infancy. J Bone Joint Surg [Am]1978;60-A:679-80.

9. Kristensen G, Neilsen K, Blyme PJ. Snapping knee from biceps femoris tendon: a case report. Acta Orthop Scand 1989;60:621.

10. Lokiec F, Velkes S, Schindler A, Pritsch M. The snapping biceps femoris syndrome. Clin Orthop 1992;283:205-6.

11. Lyu SR, Wu JJ. Snapping syndrome caused by semitendinosus tendon: a case report. J Bone Joint Surg [Am] 1989;71-A:303-5.

12. Torisu T, Nakamura Y, Kataoka M, Izumi H. Intra-articular rheumatoid nodules of the knee joint. Ryumachi 1990;30:85-9.

13. Kartus J, Movin T, Karlsson J. Donor-site morbidity and anterior knee problems after anterior cruciate ligament reconstruction using autografts. Arthroscopy 2001;17:971-80 